\title{
Reliability Evaluation under Shock Model
}

\author{
Emmanuel Ejembi ${ }^{1}$ and Shawulu Hunira $\operatorname{Nggada}^{2}$ \\ ${ }^{1}$ Department of Computer Science, Faculty of Computing and Informatics, \\ Namibia University of Science and Technology, 13 Storch Street, \\ Windhoek, Namibia \\ ${ }^{2}$ Computer Information Science, Higher Colleges of Technology, Ras Al Khaimah \\ Women's Campus, Ras Al Khaimah, United Arab Emirates \\ 1eejembi@nust.na, ${ }^{2}$ snggada@hct.ac.ae
}

\begin{abstract}
The approximation of reliability value for an engineering system is significant to predicting its possible successful operation. Typically, such approximation is done by taking into account system attributes and, or factors that may influence the operation of the system. An example of such attribute is calendar age while an example of a factor that could influence system operation is shock. This paper investigates the effect of shock on the reliability of a system component and then the system in its entirety. A general shock model is introduced from where the reliability model is developed with the assumption that components and system failure follows the exponential distribution. The developed model is then evaluated on example case studies.
\end{abstract}

Keywords: Reliability, shock model, maximum damage, exponential distribution

\section{Introduction}

Safety-critical systems such as aircraft, nuclear power plants, military installations, computer-driven medical equipment etc., are characterized by high level of complexity. This could be attributed to the number of constituent components, number and types of function to perform number and types of inputs, etc. Thus, the complexity of such systems suggests that potential hazards are to be enumerated as exhaustive as possible with their effects and mitigations outlined. The analysis of such potential hazards would inform reliability and availability evaluations. Typically, the requirements for the availability and reliability of such systems are very high. The idea of a quantitative analysis of reliability dates back to about the 1940s, at which time mathematical techniques were applied to many operational and strategic problems in World War II [1]. Prior to this period, the concept of reliability was primarily qualitative and subjective, based on intuitive notions [1]. Actuarial methods had been used to estimate survivorship of railroad equipment and in other applications early in the twentieth century while extreme value theory was used to model fatigue life of materials beginning in the 1930s [1]. A more quantitative or mathematical and formal approach to reliability grew out of the demands of modern technology, and particularly out of the experiences in the Second World War with complex military systems [2]. Barlow [3] provides a historical perspective of mathematical reliability theory up to 1940s. Similar perspectives on reliability engineering in electronic equipment and on software reliability can be found in [4], [5], and [6]. Rackwitz [7] also provides a review and some prospects of reliability analysis in early years.

Pharm, [8] defined reliability as the probability of success or the probability that the system will perform its intended function under specific design limits. In more specific

Received (May 15, 2018), Review Result (July 28, 2018), Accepted (August 8, 2018) 
terms, reliability is the probability that a product or a part will operate properly for a specified period of time (design life) under the design operating conditions (such as temperature, volt, etc.,) without failure. In other words, reliability may be used as measure of the system in providing its function properly. Reliability is one of the quality characteristics that consumers require from the manufacturer of products [8].

The reliability performance of a system for a mission under various conditions is very important in many industries, for instance military and everyday life situations. Although the qualitative concept of reliability is not new, its quantitative aspects have been improved. Such development has resulted from the increasing needs for highly reliable systems and, safer and cheaper components [9].

There are several methods of improving system reliability, e.g., using large safety factors, reducing the complexity of the system, increasing the reliability of the components through a product improvement programme, using structural redundancy and practicing a planned maintenance and repair schedule.

Maintenance modeling based on calendar age has been substantially investigated [10] however, certain component may deteriorate based on shock or combination of calendar age and shock. Hence, maintenance modeling based on calendar age alone may not be sufficient for certain systems. It is likely that a system will comprise of components that deteriorate subject to calendar age, some to shock while others to both. Therefore, there is need to investigate the shock model on system reliability.

Shock is an event that causes perturbation to the system, leading to its deterioration, the effect of these shocks on the system is measured by a process called the wear or damage process [11]. For instance, the wheel of an automobile receives shock while moving. The level of shock depends on the impact with the object or road, e.g. pothole, rough road, etc. The wheel has a threshold beyond which if it receives further shock it will result into failure. This paper investigates the effect of shock model on system reliability by using existing mathematical models to further develop model for component reliability and subsequently, system reliability.

This paper is organised as follows. Section 2 discusses component and system reliability and also gives their relationships. Sections 3 discusses shock model and gives the mathematical formula representing shock. A mathematical model for deriving the reliability of a parallel system using exponential distribution is introduced in Section 4. Conclusions are drawn in Section 5.

\section{Component and System Reliability}

The reliability of a component/system is based on precisely defined concepts. Sequel to the fact that in a population of supposedly identical components operating under similar conditions the components could fail at different points in time, then a failure phenomenon can only be described in probabilistic terms. Thus, the basic definitions of reliability can also depend on concepts from probability theory. These concepts provide the basis for quantifying the reliability of a component. Component performance may degrade with time due to some factors from component themselves or environment, which may include wear, erosion, shocks which correspondingly decrease component reliability. Component may also suddenly fail due to excessive loading, or some other related reasons.

The main strenuous task of a systems engineer is to evaluate several reliability parameters of a given system. The system configuration may differ from simple (consisting of a fewer components) to complex (consisting of hundreds of components). One method for analysing such systems is to disintegrate them into subsystems of opportune size, each representing a precise function. Reliabilities of all the subsystems are evaluated and then integrated to find the reliability of the entire system, utilising certain probability laws. However, this approach requires 
total information about the physical structure of the system. For all models, the assumption is that each component fails independently, i.e., the failure of any component does not alter the failure of the rest of the components.

There are several models proposed by researchers to evaluate system reliability, these models include time dependent hazard, Gamma, stress dependent hazard, Markov, Weibull distribution, Exponential distribution, Duane's and Bayesian, Poisson distribution, etc. [12].

The exponential distribution is widely used compared to other distribution because of it constant failure rate and simplicity, and thus, plays an important role in system reliability especially when maintenance is not taken into account. The lifetimes of electronic and electrical components and systems have been modeled using the exponential distribution. This distribution is good when a used component that has not failed is as good as a new component, a rather restrictive assumption. This implies that, it must be used diplomatically since numerous applications subsist where the restriction of the memoryless property may not apply [8]

The time of failure by an exponential failure time density function was described by (Pharm, 2006). This is given as

$$
f(t)=\frac{1}{\theta} e^{\frac{t}{\theta}} \quad t \geq 0, \theta>0
$$

And this will lead to the reliability function

$$
\begin{array}{cc}
R(t)=\int_{t}^{\infty} \frac{1}{\theta} e^{\frac{t}{\theta}} d t=e^{\frac{-t}{\theta}} & t \geq 0 \\
f(t)=\frac{1}{\theta} e^{\frac{-t}{\theta}}=\lambda e^{-\lambda t}, & t \geq 0 \\
R(t)=e^{\frac{-t}{\theta}}=e^{-\lambda t}, & t \geq 0
\end{array}
$$

Where $\theta=1 / \lambda>0$ is mean time to failure (MTTF) parameter and $\lambda \geq 0$ is a constants failure rate (Pharm, 2006).

The hazard function or failure rate for the exponential distribution on density function is constant. i.e.,

$$
h(t)=\frac{f(t)}{R(t)}=\frac{\frac{1}{\theta} e^{-\frac{i}{\theta}}}{e^{-\frac{1}{\theta}}}=\frac{1}{\theta}=\lambda
$$

The exponential distribution is an excellent model for the long flat "intrinsic failure" portion of the bathtub curve since most components and system spend some of their life time in this portion of the bathtub curve, and this justifies the frequent use of the exponential distribution (where early failure or wear out is not of concern) [8].

\section{Shock Model}

According to Rangan and Tansu [13], shocks are events which cause perturbation to the system, leading to its deterioration and consequent failure. The effect of these shocks on the system is measured by a process called wear or damage process. The wear process is denoted by $\{\mathrm{D}(\mathrm{t}): \mathrm{t} \geq 0\}$, which is represented as the deterioration level or cumulative damage level at time $t$. The shock arrives at random time which are described by the associated counting process $\{\mathrm{N}(\mathrm{t}): \mathrm{t} \geq 0\}$. A system will fail when the level of damage $\mathrm{D}(\mathrm{t})$ exceeds a damage threshold. A shock that leads to threshold crossing is referred to as lethal shocking [13].

Yehand Zhang [14] introduced a class of shock models and called them $\delta$-shock models. The early shock models concentrated solely on the magnitude of the damage 
caused by the shocks which generate attention to the frequency of the shocks. Thus, in a $\delta$-shock model, a shock is a fatal shock if the time elapsed from the previous shock to the current shock is less than the specified value $\delta$, hereafter referred to as the threshold value, and the system fails at the time of the occurrence of the fatal shock. This process is more practical because the cumulative damage process is abstract and usually not physically observable. In addition, system may not withstand successive shocks at short intervals. For instance, elastic material will stretch on the application of a shock and will take time to recover. Any further shock before the recovery is complete will make the material to break. In view of the relevance of this class of models in real systems, it seems worthwhile to make a comprehensive analysis of such a modeling approach [14].

A system suffers some damage due to shocks and the amount of shock impacted is cumulative, it fails when the total damage has exceeded it threshold, and the failure in time is obtain by cumulative process. However, it might be impossible to estimate the occurrence of shocks and the total damage occurred on the system at each shock [15].

Consider a system that is subjected to shocks and suffers some damage at each shock. Let $\mathrm{W}_{\mathrm{j}}(\mathrm{j}=1,2, . ., \mathrm{m})$ denotes the damage produced by the jth shock, $\mathrm{m}$ being the maximum number of shocks experienced by the component, where $\mathrm{W}_{0}=0$. Let $\mathrm{N}(\mathrm{t})$ denote the total number of shocks up to time $t(t \geq 0)$. The total damage is given as follows.

$$
Z(t)=\sum_{j=0}^{N(t)} W_{j} \quad ;(N(t)=0,1,2, . ., m)
$$

Where $Z(t)$ represent the total damage at time $t$. It is assumed that the system fails when the total damage at time $t$ has exceeded a pre-specified level for the first time [15].

\section{Reliability Modeling under Shock Model}

The various events that can cause shocks to engineering system have been reviewed in the previous chapter. Kodo and Nakagawa [15] compared three cumulative damage models of shocks while Ghorbani [16] investigated the impact of degradation process and random shocks on systems and determined the optimal maintenance policies for such systems. Tang and Lam [17] studied a $\delta$-shock maintenance model for a deteriorating system, on the assumption that shocks arrived according to a renewal process and the inter-arrival time of shocks has a Weibull distribution or gamma distribution. Some more general shock models were studied by Barlow and Proschan [18]. A proposed model to evaluate the reliability of multicomponent degradation systems suffering two kinds of competing failure causes, which are internal degradation process and damage from external shocks, was investigated by Liu et al., [19]. Li et al., [20] studied the reliability distribution of an individual component subject to multiple degradation processes and random shocks.

From literature, the inter-arrival time $\mathrm{t}$ is usually taken as the independent variable and the reliability $\mathrm{R}(\mathrm{t})$ is treated as a random variable. The connection of $\mathrm{R}(\mathrm{t})$ to shock is relevant to system reliability. Causality principle demands that a response to damage exists only if there is a cause. It is not straight forward to assume that time is the cause of shock. Other factors such as friction, uneven line of motion, etc., may cause shock. Additionally, when shock occurs it causes damage to a certain level that is proportional to the magnitude of the shock.

\subsection{Derivation of Shock-Damage Model}

Assumptions of the Model 
i This paper proposes that the variation of damage level $\mathrm{D}$ with respect to shock $\mathrm{S}$ is directly proportional to the sum of the damage level $\mathrm{D}_{0}$ that already exists and D due to the current shock.

ii. A component or a system fails completely after it has reached a maximum damage level $D_{m}$ having received the maximum number of shocks Sm.

iii. The damage levels caused by individual shock are independent

iv The sensitivity parameter $\beta, D_{m}$ and $S m$ may or may not be the same for different components.

Assumption (i) above can be expressed mathematically as:

$$
\frac{d D}{d S} \alpha\left(D_{0}+D\right)
$$

This implies that,

$$
\begin{gathered}
\frac{d D}{d S}=\beta\left(D_{0}+D\right) \text { or } \\
\frac{d D}{\left(D_{0}+D\right)}=\beta d S
\end{gathered}
$$

One time integration of equation 8 gives:

$$
\begin{aligned}
& \operatorname{In}\left(D_{0}+D\right)=\beta S+k \\
& \left(D_{0}+D\right)=e^{\beta s+k}=e^{\beta s} \cdot e^{k}=A e^{\beta s} \\
& D(s)=A e^{\beta S}-D_{0}
\end{aligned}
$$

The arbitrary constant $\mathrm{A}$ in equation 9 is determined by the requirement that in the absence of shock $\mathrm{D}(0)=\mathrm{D}_{0}$; i.e.,

$$
D_{0}=A e^{\beta \cdot 0}-D_{0}=A-D_{0}
$$

This implies that $A=2 D_{0}$

Therefore

$$
D(s)=2 D_{0} e^{\beta s}-D_{0}=D_{0}\left(2 e^{\beta S}-1\right) ; \mathrm{D}_{0}>0
$$

Let $\mathrm{D}_{\mathrm{m}}$ be the maximum level of damage at which the component/system fails completely and let this occur after $S_{\mathrm{m}}$ total number of shocks (assumption ii). This means

$$
D(s)=D_{0}\left(2 e^{\beta S}-1\right) ; \quad 0 \leq \mathrm{S} \leq \mathrm{S}_{\mathrm{m}}
$$

And

$$
D_{m}=D_{0}\left(2 e^{\beta S_{m}}-1\right)
$$

$\mathrm{D}(\mathrm{s})$ can be normalised to a probability distribution as:

$$
\int_{0}^{S_{m}} B D(s) d s=1
$$

Where $\mathrm{B}$ is the normalizing factor.

Therefore,

$$
B D_{0} \int_{0}^{S_{m}}\left(2 e^{\beta S}-1\right) d s=1
$$

By integration, equation 10 becomes:

$$
\begin{aligned}
& B D_{0}\left[\frac{2}{\beta} e^{\beta S}-S\right]_{0}^{S_{m}}=1 \\
& B D_{0}\left[\frac{2}{\beta} e^{\beta S_{m}}-S_{m}-\frac{2}{\beta}\right]=1
\end{aligned}
$$

Solving for B gives: 
Hence,

$$
B=\frac{1}{D_{0}\left[\frac{2}{\beta} e^{\beta S_{m}}-S_{m}-\frac{2}{\beta}\right]}
$$

$$
D_{n}(s)=\frac{e^{\beta S}-1}{\frac{2}{\beta} e^{\beta S_{m}}-S_{m}-\frac{2}{\beta}}
$$

$\mathrm{D}_{\mathrm{n}}(\mathrm{s})$ in equation 12 is regarded as the damage probability distribution of the component/system, $\beta$ is the reliability sensitivity parameter after $S_{k}(k<m)$ number of shocks. The damage failure level $\mathrm{F}\left(\mathrm{S}_{\mathrm{k}}\right)$ is given by:

i.e.,

$$
F\left(s_{k}\right)=\int_{0}^{S_{k}} D_{n}(s) d s
$$

$$
\begin{aligned}
& F\left(s_{k}\right)=\frac{1}{N} \int_{0}^{S_{k}}\left(2 e^{\beta S}-s\right) d s \\
& F\left(s_{k}\right)=\frac{1}{N}\left[\frac{2}{\beta} e^{\beta S}-S\right]_{0}^{S_{k}} \\
& F\left(s_{k}\right)=\frac{1}{N}\left[\frac{2}{\beta} e^{\beta S_{k}}-S_{k}-\frac{2}{\beta}\right]
\end{aligned}
$$

Where $\mathrm{N}$ is the denominator of the normalised damage distribution, which is given as:

$$
\mathrm{N}=\left[\frac{2}{\beta} e^{\beta S_{m}}-S_{m}-\frac{2}{\beta}\right]
$$

\subsection{Component Reliability under Shock Model}

The component of an engineering system may undergo multiple numbers of shock during operation. Therefore, after multiple numbers of damage due to shocks received, the cumulative failure distribution for each component is obtained using equation 14. From Ebeling [21], the reliability of each component becomes;

$$
\begin{aligned}
& R_{1}\left(s_{k}\right)=1-F_{1}\left(s_{k}\right), \\
& R_{2}\left(s_{k}\right)=1-F_{2}\left(s_{k}\right), \\
& R_{3}\left(s_{k}\right)=1-F_{3}\left(s_{k}\right), \\
& \cdot \\
& \cdot \\
& R_{i}\left(s_{k}\right)=1-F_{i}\left(s_{k}\right)
\end{aligned}
$$

Where $i$ refers to the ith component, $0<i \leq n, n$ is the total number of component in the system, $F_{i}\left(s_{k}\right)$ is the failure damage distribution of the ith component $R_{i}\left(s_{k}\right)$ is the reliability corresponding to number of shock $S_{k}$ of the ith component.

\subsection{System Reliability under Shock Model}

In a parallel system structure, an active redundant system consisting of four components which are connected parallel to each other is considered in this paper as shown in Figure 1 below. 


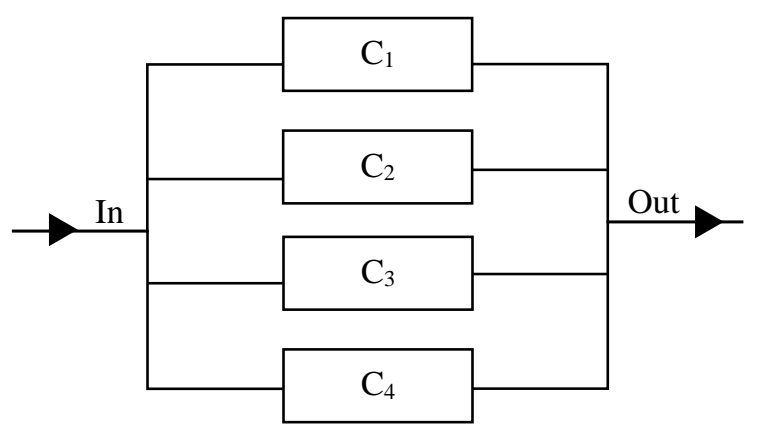

Figure 1. A Parallel System

The damage failure level $\mathrm{F}\left(\mathrm{S}_{\mathrm{k}}\right)$ for each component is already established in equation 14 as found in Ebeling [21]. Subsequently, the reliability of a component is established in equation 16. The redundant system in Figure 1 will fail when all the components reach their maximum damage level $\mathrm{D}_{\mathrm{m}}$. Hence, the reliability of the system under shock model could be obtained as follows using assumption (iii) [21].

$$
R_{s}\left(S_{k}\right)=1-\left(1-R_{1}\left(S_{k}\right)\right) \cdot\left(1-R_{2}\left(S_{k}\right)\right) \cdot\left(1-R_{3}\left(S_{k}\right)\right) \cdot\left(1-R_{4}\left(S_{k}\right)\right)
$$

Where $R_{s}\left(S_{k}\right)$ is the combine reliability of the entire component, corresponding to number of shock $S_{\mathrm{k}}$. Equation 17 is transformed into its generalised form as shown in equation 18.

$$
R_{S}\left(S_{k}\right)=1-\prod_{i=1}^{m}\left(1-R_{i}\left(S_{k}\right)\right)
$$

Where $i$ refers to the ith component and $m$ is the total number of components in the system.

\section{Model Evaluations}

The developed model of equation 16 for component reliability under shock model and that of system reliability as seen in equation 18 are evaluated in this section to demonstrate their applicability.

\subsection{Component Level Evaluation}

Typically, an engineering system comprises of more than one component. Their respective reliabilities could be evaluated using equation 16 . When the magnitude of shocks impacted on each component varies, their respective cumulative failure distribution $\mathrm{F}\left(\mathrm{s}_{\mathrm{k}}\right)$, could be evaluated using equation 14 .

The results for the evaluation of the component reliability under shock model using modified exponential distribution is shown in Table 1. These results were obtained for various values of the sensitivity parameter to demonstrate the effect it has on reliability. The sensitivity parameter values were arbitrarily chosen, however, its value has to be between 0 and 1 . The number of shocks considered is 1000. Due to space limitation, it is impossible to show the component reliability for shock step of 1 unit, therefore a shock step of 50 units is considered. This implies that the shock sequence is $0,50,150,200$, $250 \ldots . . . .1000$. 
Table 1. Component Reliability

\begin{tabular}{|l|l|l|l|l|}
\hline \multirow{2}{*}{$\begin{array}{l}\text { Shock } \\
\left(\mathrm{S}_{\mathrm{k}}\right)\end{array}$} & \multicolumn{4}{|l}{$\begin{array}{l}\text { Component Reliability } \\
\mathrm{R}_{\mathrm{j}}\left(\mathrm{S}_{\mathrm{k}}\right)\end{array}$} \\
\cline { 2 - 5 } & $\begin{array}{l}\text { Comp 1 } \\
\beta=0.001\end{array}$ & $\begin{array}{l}\text { Comp 2 } \\
\beta=0.004\end{array}$ & $\begin{array}{l}\text { Comp 3 } \\
\beta=0.007\end{array}$ & $\begin{array}{l}\text { Comp 4 } \\
\beta=0.01\end{array}$ \\
\hline 0 & 1.0000 & 1.0000 & 1.0000 & 1.0000 \\
\hline 50 & 0.9802 & 0.9979 & 0.9998 & 1.000 \\
\hline 100 & 0.9567 & 0.9947 & 0.9994 & 0.9999 \\
\hline 150 & 0.9309 & 0.9903 & 0.9988 & 0.9999 \\
\hline 200 & 0.9021 & 0.9845 & 0.9979 & 0.9998 \\
\hline 250 & 0.8720 & 0.9771 & 0.9966 & 0.9996 \\
\hline 300 & 0.8387 & 0.9675 & 0.9947 & 0.9992 \\
\hline 350 & 0.8027 & 0.9554 & 0.9917 & 0.9987 \\
\hline 400 & 0.7637 & 0.9403 & 0.9875 & 0.9978 \\
\hline 450 & 0.7217 & 0.9213 & 0.9816 & 0.9962 \\
\hline 500 & 0.6670 & 0.8322 & 0.9730 & 0.9937 \\
\hline 550 & 0.6279 & 0.7327 & 0.9608 & 0.9895 \\
\hline 600 & 0.5199 & 0.6652 & 0.9435 & 0.9826 \\
\hline 650 & 0.5199 & 0.5823 & 0.9187 & 0.9712 \\
\hline 700 & 0.4601 & 0.7327 & 0.8836 & 0.9524 \\
\hline 750 & 0.3962 & 0.6652 & 0.8336 & 0.9213 \\
\hline 800 & 0.3280 & 0.5823 & 0.7627 & 0.8702 \\
\hline 850 & 0.2553 & 0.4807 & 0.6617 & 0.7858 \\
\hline 900 & 0.1777 & 0.3588 & 0.5189 & 0.6407 \\
\hline 950 & 0.0951 & 0.2035 & 0.3159 & 0.4174 \\
\hline 1000 & 0.0055 & 0.0166 & 0.0277 & 0.0160 \\
\hline & & & & \\
\hline
\end{tabular}

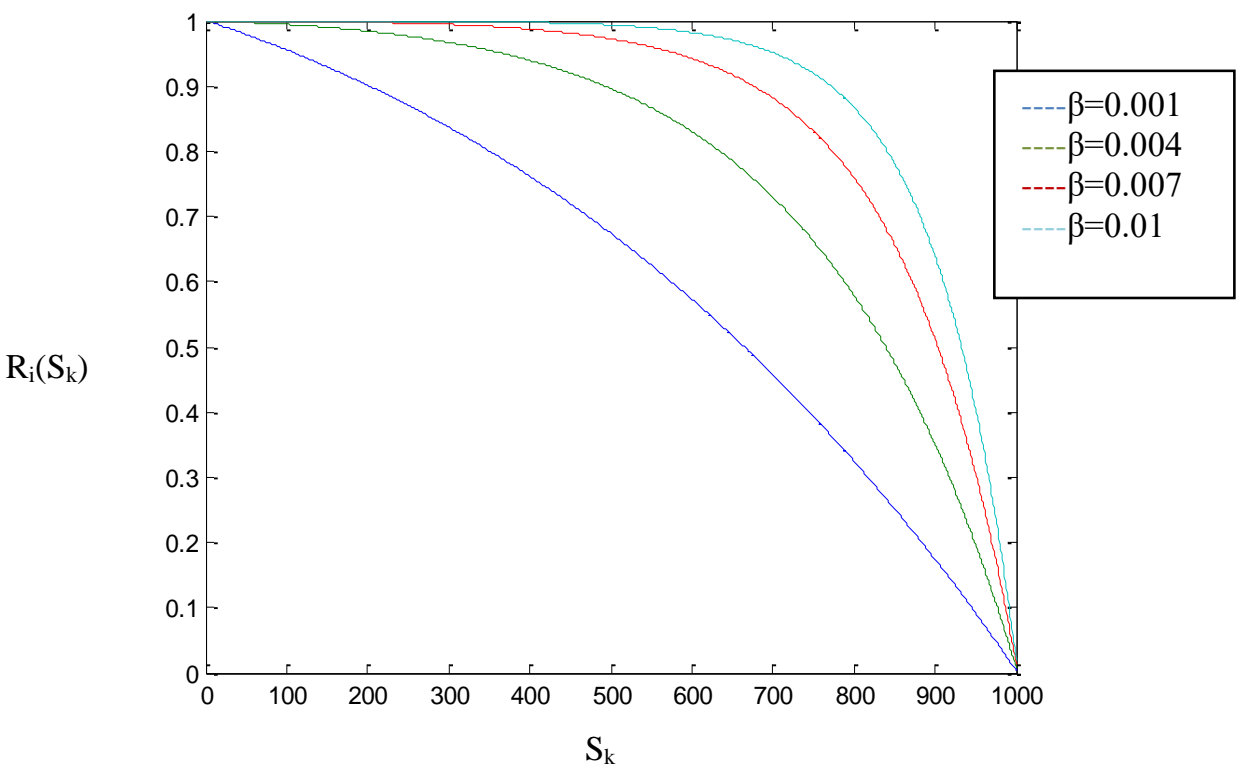

Figure 2. Components Reliability

Figure 2 shows the component reliability $\mathrm{R}_{\mathrm{j}}\left(\mathrm{s}_{\mathrm{k}}\right)$ of different sensitivity parameters, with respect to shock $\mathrm{S}_{\mathrm{k}}$. Components $\mathrm{C}_{1}, \mathrm{C}_{2}, \mathrm{C}_{3}$ and $\mathrm{C}_{4}$ has sensitivity parameter as 0.001 , $0.004,0.007$ and 0.01 respectively. From the graph it could be observed that as each 
active component undergoes more shock the damage caused by the shock increases. The component reliability $R_{i}\left(S_{k}\right)$ decrease with increase in shock $S_{k}$.

From the assumption made, the component fails completely after it has reached a maximum damage level $D_{m}$, having received a maximum number of shocks, $S_{m} . S_{m}$ is assumed to be 1000 shock, the sensitivity parameter $\beta$ was assumed to have varying values for each of the components.

Component 1 which has the least sensitivity parameter is likely to be the first to experience failure after reaching its maximum shock $S_{\mathrm{m}}$ compared to 2,3 and 4 . The sequence of failure after component 1 would then be component 2,3 and lastly 4 .

Therefore, from the graph the reliability of the components increases with increase in sensitivity parameter $\beta$. The sharp drop in the curve of each component infers that the component is close to failure before reaching its maximum shock $S_{\mathrm{m}}$.

The magnitude of individual shock has an effect on the components. The shock magnitude increases the accumulated damage created on it. When the component is closer to it maximum damage level $\mathrm{D}_{\mathrm{m}}$, any small amount of shock may have a greater impact on the component reliability, and this may cause a fast drop in the reliability.

\subsection{System Level Evaluation}

For a multi-component system with m components configured in parallel, assumption (iii) enables the use of two terms prescription for the total reliability of the system as in equation 18. Hence, the reliability for the system as used in this paper is evaluated using equation 18. The results of the evaluation are shown in Table 2 and the graph representation is shown in Figure 3.

Table 2. System Reliability

\begin{tabular}{|l|l|}
\hline $\begin{array}{l}\text { Shock } \\
\left(\mathrm{S}_{\mathrm{k}}\right)\end{array}$ & $\begin{array}{l}\text { System } \\
\text { Reliability } \\
\mathrm{R}_{\mathrm{S}}\left(\mathrm{S}_{\mathrm{k}}\right)\end{array}$ \\
\hline 0 & 1.0000 \\
\hline 50 & 1.0000 \\
\hline 100 & 1.0000 \\
\hline 150 & 1.0000 \\
\hline 200 & 1.0000 \\
\hline 250 & 1.0000 \\
\hline 300 & 1.0000 \\
\hline 350 & 1.0000 \\
\hline 400 & 1.0000 \\
\hline 450 & 1.0000 \\
\hline 500 & 1.0000 \\
\hline
\end{tabular}

\begin{tabular}{|l|l|}
\hline $\begin{array}{l}\text { Shock } \\
\left(\mathrm{S}_{\mathrm{k}}\right)\end{array}$ & $\begin{array}{l}\text { System } \\
\text { Reliability } \\
\mathrm{R}_{\mathrm{S}}\left(\mathrm{S}_{\mathrm{k}}\right)\end{array}$ \\
\hline 550 & 1.0000 \\
\hline 600 & 0.9999 \\
\hline 650 & 0.9998 \\
\hline 700 & 0.9973 \\
\hline 750 & 0.9973 \\
\hline 800 & 0.9911 \\
\hline 850 & 0.9713 \\
\hline 900 & 0.9076 \\
\hline 950 & 0.7060 \\
\hline 1000 & 0.0450 \\
\hline
\end{tabular}




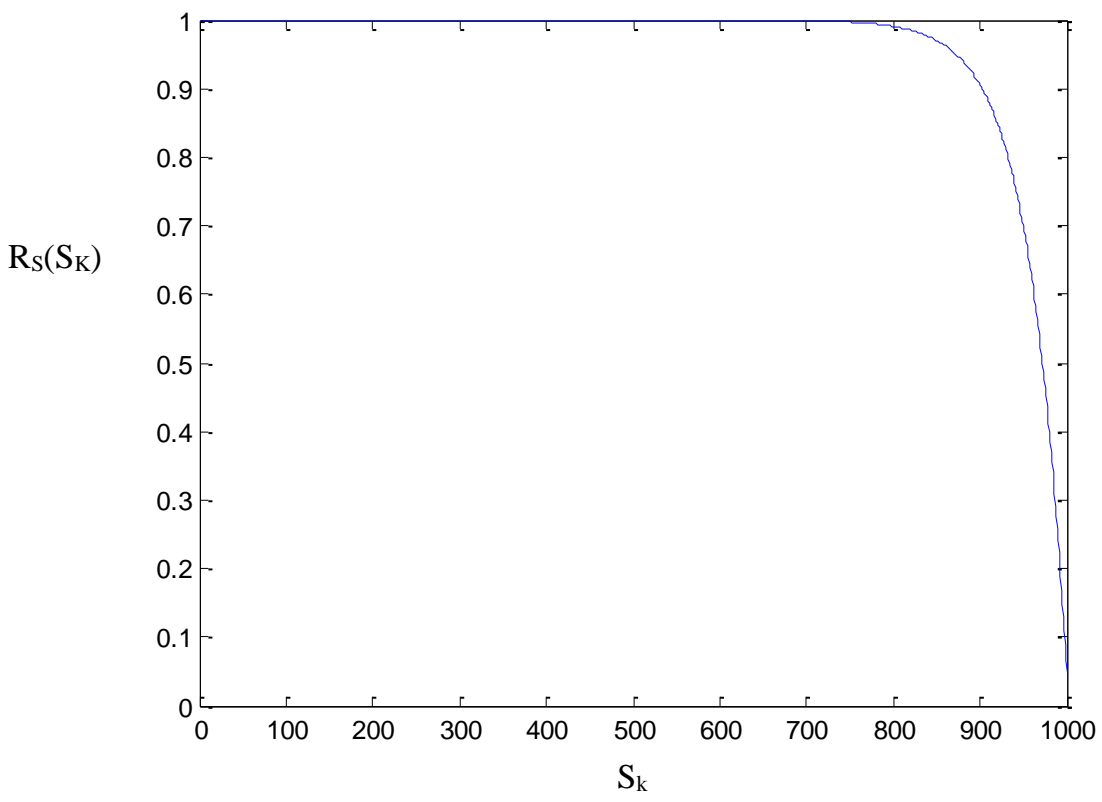

Figure 3. Total Reliability of the Four Components (System Reliability)

Figure 3 shows the resultant reliability of the four components configured in parallel, with respect to shock. This gives the system reliability. The sensitivity value used for each component was the same as that found in Figure 2. The system reliability in Figure 3 is higher than the components reliability in Figure 2. This implies that for an active redundant system, if one component attains its maximum damage level, the system will continue to function effectively but has a slight drop in reliability. The system fails completely when the entire components reach their maximum damage level.

\section{Conclusions}

In this paper, the effect of shock on component reliability was investigated. A model to evaluating the reliability was then mathematically established based on other established models found in literature. The modeling of component reliability is of significance since system reliability could be evaluated using individual component failure data and reliability evaluation model. Thus, a model to evaluating system reliability was also established. The system reliability that was modeled in this paper is focused on a parallel system. This does not imply that the developed component reliability could not be used to establishing system reliability for series system or a system that comprise of both series and parallel configurations. To demonstrate the applicability of the models, individual component reliabilities for four different components were evaluated with varying failure data. Similarly, the reliability of a system comprising of the four components configured in parallel was evaluated. In general, the failure characteristics of the components were assumed to follow the exponential distribution. The results obtained show that reliability decreases with increased shock. This pattern was expected and thus, the evaluations performed demonstrated the sufficiency of the established models under shock and exponential distribution. However, further work will be required to investigate the effects of maintenance on shock. 


\section{References}

[1] W. R. Blischke and M. Probhakar, "Case Studies in Reliability and Maintenance", John Wiley \& Sons, INC., Publication, USA, (2003), pp. 1-32.

[2] R. F. Barlow and F. Proschan, "Mathematical Theory of Reliability", Wiley, New York, (1965), pp. 1620.

[3] R. E. Barlow, "Mathematical Theory of Reliability: A Historical Perspective", IEEE Transactions on Reliability, vol. R-33, no. 1, (1984), pp. 16-20.

[4] A. Coppola, "Reliability Engineering of Electron Equipment: A Historical Perspective", IEEE Transactions on Reliability, (1984), pp. 33:29-35.

[5] J. G. Shanthikumar, "Software Reliability Models: A Review", Microelectronics Reliability, vol. 23, no. 5, (1983), pp. 903-943.

[6] M. L. Shooman, "Software reliability: A historical perspective", IEEE Transactions on Reliability, vol. R-33, no. 1, (1984), pp. 48-55.

[7] R. Rackwitz, "Reliability analysis-a review and some perspective", Structural Safety, vol. 23, no. 4, (2001), pp. 365-395.

[8] H. Pharm, "System Software Reliability: Springer Series in Reliability Engineering, Department of Industrial Engineering Rutgers", The State University of New Jersey, USA, (2006), pp. 440-451.

[9] A. T. Frank and C. L. Hwang, "Optimization Techniques for System Reliability with Redundancy - A Review”, IEEE Transaction on Reliability, vol. R-26, no. 3, (1977), pp. 148-155.

[10] S. H. Nggada, "Characteristics of Exponential Distribution with Respect to Preventive Maintenance", International Journal of Control and Automation, ISSN: 2005-4297, vol. 8, no. 1, (2015), pp. 179-188.

[11] A. Rangan and Y. Sarada, "Optimal Replacement of Systems Subject to Shocks and Random Threshold Failure”, International Journal of Quality and Reliability Management, vol. 23, (2006), pp. 1176-1191.

[12] P. Hoang, "Handbook of Reliability Engineering", Rutgers University Piscataway, Springer, ISBN 1852334533, New Jersey, USA, (2003).

[13] A. Rangan and A. Tansu, "A New Shock Model for System Subject to Random Threshold Failure", International Journal of Mechanical Aerospace, Industrial, Mechatronics and Manufacturing Engineering, vol. 2, no. 11, (2008), pp. 1267-1272.

[14] Y. Lam and Y. L. Zhang, "A Shock Model for the Maintenance Problem of a Repairable System", Computer and Operational Research, vol. 31, (2004), pp. 1807-1820.

[15] I. Kodo and N. Toshio, "Comparison of Three Cumulative Damage Models", Quality Technology and Quantitative Management, vol. 8, no. 1, (2011), pp. 57-66.

[16] S. Ghorbani, "Reliability Analysis for System Subject to Degradation and Shocks", Rutgers, The State University of New Jersey, (2014), pp. 21-71

[17] Y. Y. Tang and Y. Lam, “A $\delta$-Shock Maintenance Model for a Deteriorating System”, European Journal of Operational Research, vol. 168, (2006), pp. 541-556.

[18] R. E. Barlow and F. Proschan, "Statistical Theory of Reliability and Life Testing: Probability Models", New York: Rinehart and Winston, (1975a), pp. 1458-1467.

[19] Y. Liu, H. Z. Huang and H. Pham, "Reliability Evaluation of Systems with Degradation and Random Shocks", Reliability and Maintainability Symposium (RAMS), (2008), pp. 328-333.

[20] C. Y. Li, X. Chen, X. S. Yi and J. Y. Tao, "Degraded Systems with Multiple Performance Parameter Subject to Shocks", Reliability and Maintainability Symposium (RAMS) Proceeding -Annual, (2010), pp. 1-6.

[21] C. E. Ebeling, “An Introduction to Reliability and Maintainability Engineering”, McGraw-Hills, USA, (1997).

\section{Authors}

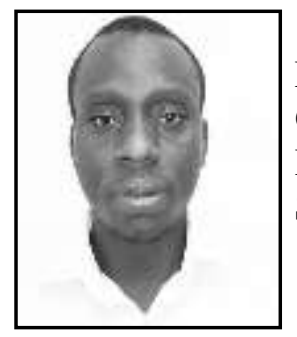

Ejembi Emmanuel has BSc Physics from Benue State University, Makurdi - Nigeria, Diploma in Computer Operator and Certificate in Computer Maintenance both from Orbit's Computer Investment, Benue State, Nigeria. He pursued a Masters degree in Computer Science at Namibia University of Science and Technology. 


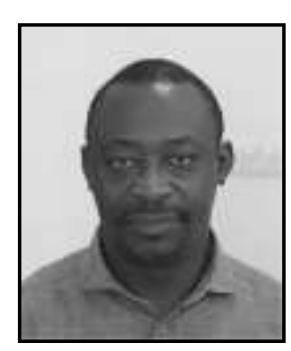

Shawulu Hunira Nggada has $\mathrm{PhD}$ in Computer Science and PGDip in Research Training both from University of Hull - UK, MSc Software Engineering from University of Bradford - UK and B. Tech (Hons) in Computer Science from Abubakar Tafawa Balewa University, Nigeria. He has Chartered IT Professional membership of BCS The Chartered Institute for IT, Chartered Engineer membership of Engineering Council - UK. He is a Senior Member of IEEE. He is currently a Faculty at Higher Colleges of Technology, UAE. He previously held a Faculty position at Namibia University of Science and Technology. 